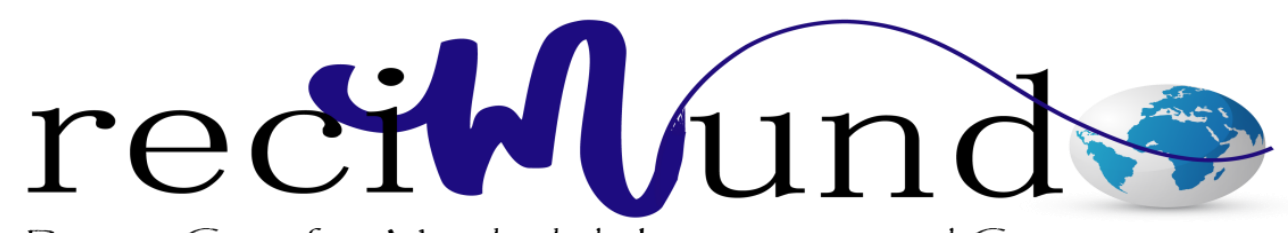

Revista Científica Mundo de la Investigación y el Conocimiento

Fernando Eduardo Viteri Luque ${ }^{\text {a, }}$ Luis Alemán Herrera Lozano ${ }^{\text {b; Antonio }}$ Fernando Bazurto Quiroz $^{\mathrm{c}}$

Las Tendencias del Marketing: Cuales son y definiciones

Revista Científica Mundo de la Investigación y el Conocimiento. Vol. 1 núm., 5, diciembre, 2017, pp. 974-988

DOI: $10.26820 /$ recimundo/1.5.2017.974-988

Editorial Saberes del Conocimiento

I. Universidad de Guayaquil; fernando.viteril@ug.edu.ec

II. Universidad de Guayaquil; luis.herreral@ug.edu.ec

III. Universidad de Guayaquil; antonio.bazurtoq@ug.edu.ec 


\section{Las Tendencias del Marketing: Cuales son y definiciones}

Vol. 1, núm. 5., (2017)

Fernando Eduardo Viteri Luque; Luis Alemán Herrera Lozano; Antonio Fernando Bazurto

Quiroz

\section{RESUMEN}

En término generales, el marketing es un conjunto de actividades destinadas a satisfacer las necesidades y deseos de los mercados meta a cambio de una utilidad o beneficio para las empresas u organizaciones que la ponen en práctica; razón por la cual, nadie duda de que el marketing es indispensable para lograr el éxito en los mercados actuales. Por ese motivo, resulta indispensable que todas las personas que son parte (directa o indirectamente) del área comercial de una empresa u organización, conozcan a profundidad cuál es la definición de marketing y sus aplicaciones (Ph.D McCarthy E., 2001).

A su vez el marketing es una disciplina que ha progresado en las últimas décadas, desarrollando nuevos enfoques que dan respuesta a las necesidades de los clientes actuales, que son cada día son más demandante. A este proceso de evolución que ha sufrido el mercadeo, es lo que se conoce como nuevas tendencias. Siendo este el punto principal del presente artículo, debido a que se aspira dejar claro cuales son las tendencias más importantes a aplicar en el entorno altamente competitivo donde se desenvuelven las organizaciones, al mismo tiempo que se pretende clarificar las definiciones de los nuevos enfoques que han surgido.

Palabras claves: marketing, tendencias, consumidor, satisfacción del cliente. 


\title{
Las Tendencias del Marketing: Cuales son y definiciones
}

Vol. 1, núm. 5., (2017)

Fernando Eduardo Viteri Luque; Luis Alemán Herrera Lozano; Antonio Fernando Bazurto Quiroz

\begin{abstract}
In general terms, marketing is a set of activities designed to satisfy the needs and desires of the target markets in exchange for a profit or benefit for the companies or organizations that put it into practice; Which is why, nobody doubts that marketing is essential to achieve success in today's markets. For this reason, it is essential that all people who are part (directly or indirectly) of the commercial area of a company or organization, know in depth what is the definition of marketing and its applications (Ph.D McCarthy E., 2001).

In turn, marketing is a discipline that has progressed in recent decades, developing new approaches that respond to the needs of current customers, who are becoming more demanding each day. This process of evolution that has undergone marketing, is what is known as new trends. This being the main point of this article, since it is hoped to make clear which are the most important trends to be applied in the highly competitive environment in which the organizations operate, while at the same time trying to clarify the definitions of the new approaches that have been developed. emerged.
\end{abstract}

Keywords: marketing, trends, consumer, customer satisfaction. 


\section{Las Tendencias del Marketing: Cuales son y definiciones}

Vol. 1, núm. 5., (2017)

Fernando Eduardo Viteri Luque; Luis Alemán Herrera Lozano; Antonio Fernando Bazurto

Quiroz

\section{Introducción.}

El enfoque del marketing ha ido evolucionando a lo largo de estos últimos años, ya la premisa dejo de ser vender a toda costa y sin que en ese momento importase el cliente, al contrario, en la actualidad las empresas están abocadas a que el cliente tenga una participación activa, amplia y en algunos casos individualizada con la organización, para lo cual el tiempo es un factor determinante al momento de establecer relaciones duraderas con los consumidores y usuarios de un servicio o producto, pues la decisión del cliente de establecer una relación de intercambio comercial con una marca determinada requiere un proceso de diálogo constante para que ambos actores (empresa y cliente) construyan un vinculo que permita la fidelización.

Los diálogos suministran una información que es más íntima y más potente que lo que podría ser cualquier registro de transacciones. Sin embargo, cada dialogo con un cliente es una oportunidad de estructurar el alcance de la relación con ese cliente- todos los ítems de conocimientos extraídos de un dialogo- pueden usarse para garantizar una relación más firme, más productiva y duradera (Negocios, n.ed).

En virtud de lo anterior, el marketing surge para dar respuesta de como establecer ese diálogo entre empresa y cliente, partiendo desde los fundamentos tradicionales hasta las nuevas tendencias en la materia que hacen más dinámica e interactivo el proceso, ya que el soporte en medio tecnológicos contribuye a cerrar las brechas existentes en materia de satisfacción y cubrir necesidades, pero para ello debe existir previamente un plan de como accionar. Es por ello que este artículo pretende dejar un precedente en cuanto a definiciones y aplicaciones de las nuevas 


\section{Las Tendencias del Marketing: Cuales son y definiciones}

Vol. 1, núm. 5., (2017)

Fernando Eduardo Viteri Luque; Luis Alemán Herrera Lozano; Antonio Fernando Bazurto Quiroz

tendencias del marketing se refieren, elementos estos que son de gran importancia para cualquier organización que aspire mantener sus indicadores de ventas y correcto manejo de marca.

\section{Materiales y métodos.}

El marketing es un proceso en el cual intervienen dos factores el cliente y la empresa, siendo una relación surge con el propósito de establecer un vínculo estrecho entre ambas partes y así generar un valor hacia el cliente. Sin embargo, en esta interacción intima entre los actores principales involucrados en un proceso de comercialización, la globalización debe ser considera y tomada en cuenta como el elemento "acelerado" dentro del proceso de cambio que, a nivel mundial, se ha venido desarrollando en todos los ámbitos humanos, pero particularmente en lo referente a lo militar, lo económico, el comercio, las finanzas, la información, la ciencia, la tecnología, el arte y la cultura (Ph.D McCarthy E., 2001).

\section{Conceptos asociados}

1.1. Necesidades, deseos y demanda: el marketing per se no crea las necesidades de los clientes, debido a que estas son inherentes a las personas, el mercadólogo lo que hace es orientar los deseos de los consumidores y estimula la demanda de determinado producto y marca a través de una seria de estrategias para tal fin (Thompson, 2006).

1.2. Valor y Satisfacción: el valor es la relación que establece el cliente entre los beneficios (funcionales, status, etc..) que percibe del producto que se ofrece y los costos (económicos, tiempo, esfuerzos) que representa adquirirlo. Otros elementos como la simplificación en la toma de decisión de compra, la lealtad y la 


\section{Las Tendencias del Marketing: Cuales son y definiciones}

Vol. 1, núm. 5., (2017)

Fernando Eduardo Viteri Luque; Luis Alemán Herrera Lozano; Antonio Fernando Bazurto

Quiroz

jerarquización de beneficios han sido incluidos en el desarrollo de la oferta, a través de la creación de propuestas de valor orientadas a configurar productos y servicios que satisfagan óptimamente las necesidades y deseos de los individuos a quienes van dirigidas (Tirado, 2013). El grado de satisfacción es el estado anímico de bienestar o decepción que se experimenta tras el uso de un bien. Y si bien la satisfacción está vinculada a criterios de comparación y expectación, donde la percepción sobre la oferta tiene un lugar muy relevante, se ha demostrado que el desempeño real de la oferta en la atención de necesidades, deseos y bienes de orden tiene un efecto más duradero y sostenible en ella, razón por la cual la mercadotecnia estratégica transita de la simbolización a la configuración de la oferta (Gutierrez, 2010).

1.3. Intercambio: implica la interacción de dos o más partes que tranzan o llegan a un acuerdo de ceder algo para obtener una cosa a cambio, estos intercambios tienen que ser mutuamente beneficiosos entre la empresa y el cliente, para construir una relación satisfactoria de largo plazo (Ph.D McCarthy E., 2001).

\section{Tendencias}

2.1. Consumidores móviles y multipantalla: Las campañas digitales se conciben para todas y cada una de las pantallas disponibles en el día a día de la audiencia (Ph.D McCarthy E., 2001). Una historia uniforme que cobra vida forma a través de contenidos interactivos optimizados en cada soporte o dispositivo. Los medios que sean capaces de responder a esta necesidad con proyectos realmente integrados serán los que crezcan considerablemente en sus ingresos (Tirado, 


\section{Las Tendencias del Marketing: Cuales son y definiciones}

Vol. 1, núm. 5., (2017)

Fernando Eduardo Viteri Luque; Luis Alemán Herrera Lozano; Antonio Fernando Bazurto Quiroz

2013). Del tiempo ocupado para ver televisión un $77 \%$ de este se hace al mismo tiempo que se utiliza otro dispositivo (Thompson, 2006).

2.2. Marketing Experiencial: Se refiere especialmente en llevar la experiencia de la marca al consumidor de una manera profunda para lograr una conexión memorable. Las experiencias son fuentes de emociones y las emociones son el motor de la toma de decisiones (Tirado, 2013).

Según (Gómez, 2013):

El marketing experiencial es el marketing de las experiencias o el marketing de las emociones, es decir, un marketing basado en provocar sentimientos y emociones en el consumidor para que éste conecte con una marca $\mathrm{n}$ un modo mucho más profundo. Ya se ha demostrado la relación entre la emoción y la compra; y como los consumidores compran más aquellas marcas que le despiertan emociones y sentimientos internos y que les hace sentir y vivir ese compromiso que los lleva a comprar esa marca.

2.3. Marketing Interactivo: Consiste en la habilidad de dirigirse a un individuo, a través del empleo de diversas técnicas de comunicación soportadas en medios tecnológicos como lo es el internet. Se diferencia del marketing digital, ya que este enfoque permite que los usuarios formen parte y se involucren en el desarrollo de campañas, siendo co-autores del proceso creativo. Es precisamente el hecho de ser "Interactivo" lo que marca la diferencia con las estrategias de mercadeo tradicional, pues por medio de esta tendencia se persigue la respuesta inmediata del consumidor (Redacción, 2008). 


\section{Las Tendencias del Marketing: Cuales son y definiciones}

Vol. 1, núm. 5., (2017)

Fernando Eduardo Viteri Luque; Luis Alemán Herrera Lozano; Antonio Fernando Bazurto

Quiroz

El marketing interactivo se presenta como un marketing inteligente y evolucionado por medio del cual se le puede ofrecer a cada cliente aquello que busca en un momento determinado, cumpliendo así con la premisa máxima de mercadeo, que es la satisfacción de los clientes. En este sentido, el proceso interactivo cumple el objetivo de segmentar cada perfil, fidelizar y en especial crear relaciones de largo plazo con los clientes (Ph.D McCarthy E., 2001).

2.4. Big Data: De acuerdo a (Negocios, n.ed) es la nueva estrategia de mercado que será indispensable para todas las empresas. La idea es manejar enormes cantidades de datos que permitan conocer patrones de información sobre las necesidades y gustos de los clientes, acercando de esta forma a las empresas a su público objetivo, es un CRM (Costumer Relationship Managament) evolucionado.

2.5. Retargeting: El retargeting (o remarketing) es una técnica de marketing digital consistente en impactar con publicidad a usuarios que previamente hayan interactuado con una organización. Esto puede significar que hayan visitado el sitio web, abierto un email o simplemente haber hecho click en un cierto enlace en algún lugar del ciberespacio. El concepto de retargeting es muy amplio, cambiante y cada vez más complejo (ONiUP, n.ed).

Del total de visitas a una tienda online, sólo un 2\% compra en su primera visita, el retargeting va a por ese $98 \%$ restante, tarea nada sencilla, ya que la clave de un buen retargeting es su capacidad de captar la mayor cantidad de porcentaje de ese $98 \%$ previamente mencionado, debido a que a veces un $0.1 \%$ o tal vez un $4 \%$, puede implicar triplicar las ventas en una organización, por lo tanto, más importante que hacer retargeting es hacerlo bien (ONiUP, n.ed).

En este sentido el retargeting aporta beneficios en: 


\section{Las Tendencias del Marketing: Cuales son y definiciones}

Vol. 1, núm. 5., (2017)

Fernando Eduardo Viteri Luque; Luis Alemán Herrera Lozano; Antonio Fernando Bazurto Quiroz

- Un ROI (retorno de la inversión) publicitario de hasta el 300\%

- Branding muy específico sin gastar mucho dinero

- Refuerzo en todo el embudo de marketing (conocimiento de marca, leads, ventas, fidelización...)

2.6. Green Marketing: Está abocado en el desarrollo y ejecución de campañas, promoción, productos, servicios y empaque de productos con componentes biodegradables que protegen el medio ambiente. Está constituido por un conjunto de acciones encaminadas al posicionamiento de una marca o producto. La meta es aumentar las ventas, pero también mejorar la imagen de la empresa (Ph.D McCarthy E., 2001). La investigación de mercados bajo la premisa de ser verde y eco amigable ha ayudado a que las tecnologías se aboquen a crear programas y modelos que permitan las mejoras dentro de la organización invirtiendo en planes de introducir tecnología que a su vez persigan la eficiencia en todos los ámbitos de la organización incluyendo aspectos ecológicos.

De acuerdo a (Merca2.0, 2014):

El eco-marketing, marketing verde y ambiental son parte de los nuevos enfoques de marketing que no se limita a modificar el enfoque, adaptar o mejorar el pensamiento, sino que busca desafiar esos enfoques y ofrecer una perspectiva sustancialmente diferente. Con más detalle, verde, ambiental y eco-marketing pertenecen al grupo de enfoques que tratan de 


\section{Las Tendencias del Marketing: Cuales son y definiciones}

Vol. 1, núm. 5., (2017)

Fernando Eduardo Viteri Luque; Luis Alemán Herrera Lozano; Antonio Fernando Bazurto

Quiroz

hacer frente a realidades ecológicas y sociales del amplio entorno del marketing.

2.7. Content Marketing: Se basa en reconocer las necesidades de los clientes y seguidores para así ofrecerles contenidos e información de interés y relevantes que cubran y satisfagan sus expectativas a través del soporte de medios tecnológicos (Redacción, Marketing de contenido, 2013). Este tipo de mercadeo debe crear un aumento de la calidad de los contenidos publicados en las redes sociales y en las estrategias de las marcas, adaptándose mejor al entorno en lugar de replicar campañas como un soporte más (Negocios, n.ed).

2.7.1. Algunos tipos de contenidos: Imágenes, infografías, vídeos, guías / tutoriales, revistas, ebooks, plantillas, whitepapers, presentaciones, aplicaciones, juegos, webinars.

2.7.2. Canales de promoción: Blogs, facebook, twitter, linkedin, Instagram, youtube, foros especializados, directorios.

\subsubsection{Beneficios del marketing de contenidos:}

Según (Nunez, 2018):

- Te une más a tu comunidad, es una forma excelente de conectar con tú público y conocerlo más a fondo.

- Te permite llegar a más personas, es decir que puedes iniciar abarcar un nuevo público. 


\section{Las Tendencias del Marketing: Cuales son y definiciones}

Vol. 1, núm. 5., (2017)

Fernando Eduardo Viteri Luque; Luis Alemán Herrera Lozano; Antonio Fernando Bazurto Quiroz

- Es una forma ideal de promocionar a una marca o los productos y servicios de la misma.

- Es una forma muy efectiva de convertir leads.

- Genera confianza entre la marca creadora de contenidos y el consumidor final. Casi siempre un usuario contento repite.

8.Storytelling: De acuerdo con (Cortés, 2015) los tiempos han cambiado y los usuarios buscan publicidad que haga sentir emociones. No basta con ofrecer publicidad no intrusiva en forma de contenidos de entretenimiento o interés informativo con relevancia para el público, ahora el consumidor pide que las marcas dominen el arte de contar historias (Nunez, 2018).

9.Gamificación: Es un término anglosajón que Sebastian Deterding definió como el uso de las mecánicas de juego en entornos ajenos al juego (Moll, 2014). El término gamificación abarca muchas disciplinas como, por ejemplo, el ámbito empresarial o la psicología y recientemente en mercadotécnica, ya que busca potenciar la motivación, la concentración, el esfuerzo, la fidelización y otros valores positivos comunes a todos los juegos. Se trata de una nueva y poderosa estrategia para influir y motivar a grupos de personas. (Cortés, 2015)

De acuerdo a (Cortés, 2015) actualmente la gamificación se está utilizando para:

- Atraer y retener audiencias y clientes a una marca.

- Animar a la gente a realizar tareas que consideran aburridas.

- Minimizar los tiempos de adopción de productos o servicios. 


\section{Las Tendencias del Marketing: Cuales son y definiciones}

Vol. 1, núm. 5., (2017)

Fernando Eduardo Viteri Luque; Luis Alemán Herrera Lozano; Antonio Fernando Bazurto

Quiroz

10.Neuromarketing: El neuromarketing estudia los procesos cerebrales de las personas y sus cambios durante la toma de decisiones con el fin de poder llegar a predecir la conducta del consumidor. El 95\% de las decisiones de compra se producen de una forma inconsciente e irracional (Cortés, 2015), debido a que el cliente/ consumidor conecta con las marcas a en el plano neuronal en tres niveles: racional, emocional e instintivo, dependiendo del grado con el que la marca satisfaga cada uno de esos niveles del cerebro, la afinidad que tenga con la marca y su deseo de compra determinará el tipo de conexión neuronal que ha efectuado y por ende la consecuencia de la interacción comercial con la organización que está ofertando sus servicios o productos.

Según (Neuromarca, 2009):

El neuromarketing consiste en la aplicación de las técnicas de investigación de las neurociencias a la investigación de marketing tradicional. A través de técnicas de medición de la actividad cerebral (como el EGG o la fMRI), las "respuestas" de los entrevistados a distintos estímulos (por ejemplo, anuncios publicitarios) son leídas directamente de su actividad cerebral. Las neurociencias permiten, por este método, averiguar qué niveles de atención está prestando los sujetos analizados a un anuncio segundo por segundo y plano por plano. De este modo se pueden tomar decisiones como por ejemplo retirar un determinado plano del anuncio final o añadir una secuencia adicional. También pueden medirse otros muchos conceptos, como la activación del sujeto o su estado emocional cuando aparece el producto 


\section{Las Tendencias del Marketing: Cuales son y definiciones}

Vol. 1, núm. 5., (2017)

Fernando Eduardo Viteri Luque; Luis Alemán Herrera Lozano; Antonio Fernando Bazurto Quiroz

en pantalla. Kevin Randall, director de estrategia de marca e investigación en Movéo Integrated Marketing, define el neuromarketing como "la práctica de usar tecnología para medir la actividad cerebral en los consumidores para utilizar esa información en el desarrollo de productos y comunicaciones" (el texto original: "the practice of using technology to measure brain activity in consumer subjects in order to inform the development of products and communications").

\section{Conclusiones.}

En los tiempos en los que se vive, donde los cambios son el día a día en el entorno mundial actual ha ocasionado que el cliente sea cada vez más exigente, de allí que el mercado comercial sea sumamente competitivo. En términos globales solo aquellas empresas que aporten verdadero valor a sus clientes en base a sólidas y rentables relaciones en el tiempo, sobrevivirán (Ph.D McCarthy E., 2001). En este sentido, surge la importancia y el interés por parte de las organizaciones en construir y fortalecer una relación a largo plazo con el cliente para lograr unas mayores tasas de rentabilidad y crecimiento sostenido.

El éxito de una organización hoy día se fundamenta en su capacidad de poner en marcha de forma exitosa las prácticas sobre los fundamentos sobre los que el marketing está basado, como reducir el coste de obtener nuevos clientes para incrementar la retención de éstos, tratar a los clientes adecuadamente, reconocer su individualidad y satisfacer sus necesidades únicas para así, asegurarse un crecimiento sostenible que le aporte altas tasas de rentabilidad, pero a su vez 


\section{Las Tendencias del Marketing: Cuales son y definiciones}

Vol. 1, núm. 5., (2017)

Fernando Eduardo Viteri Luque; Luis Alemán Herrera Lozano; Antonio Fernando Bazurto

Quiroz

aplicando nuevos enfoques más dinámicas e integrales que cumplen con el objetivo de cualquier empresa, que es generar dividendos de manera sostenida en el tiempo y para lograr esto, el cliente es el factor clave, por lo cual se establecen estrategias enfocados al último eslabón de la cadena de suministros, el consumidor.

Por ello las nuevas tendencias del marketing del siglo XXI toman fuerza y presentan un abanico de alternativas para que esta disciplina como lo es la mercadotecnia, se pueda adaptar perfectamente a las necesidades de cada empresa o en su defecto hacer un mix, creando así un enfoque personalizado y perfectamente adecuado a la realidad de la organización en un tiempo y momento determinado, que puede ser fácilmente ajustable en el futuro cercano.

\section{Referencias}

Chiavenato, I. (2001). Administración: Teoría, proceso y práctica. México: Mc Graw-Hill.

Cortés, F. (2015). Tendencias actuales del marketing. Mkt total, recuperado de: https://www.mercadotecniatotal.com/mercadotecnia/tendencias-actuales-de-marketing/.

Gómez, E. (2013). Marketing experiencial: Mejor vivirlo a que te lo cuenten. recuperado de: https://www.puromarketing.com/44/18364/experiencial-mejor-vivirlo-cuenten.html.

Gutierrez, H. (2010). Calidad total y productividad. México: McGraw Hill Interamaricana, 3era edición.

Merca2.0. (2014). Que es el Green Marketing. recuperado de: https://www.merca20.com/que-esel-green-marketing-3-definiciones/.

Moll, S. (2014). La gamificación: 7 cláves para enteder que es y como funciona. recuperado de: http://justificaturespuesta.com/gamificacion-7-claves-para-entender-que-es-y-comofuncional.

Negocios, G. y. (n.ed). Marketing relacional. Gerencia y negocios, recuperado de: http://www.degerencia.com/articulo/el_marketing_relacional_una_conceptualizacion_ne cesaria.

Neuromarca. (2009). Neuromarketing. recuperado de: http://neuromarca.com/neuromarketing/. 


\section{Las Tendencias del Marketing: Cuales son y definiciones}

Vol. 1, núm. 5., (2017)

Fernando Eduardo Viteri Luque; Luis Alemán Herrera Lozano; Antonio Fernando Bazurto Quiroz

Nunez, V. (2018). Marketing de contenidos. recuperado de: https://vilmanunez.com/que-es-elmarketing-de-contenidos/.

ONiUP. (n.ed). Que es el retargeting. recuperado de: https://oniup.com/que-es/retargeting/.

Ph.D McCarthy E., P. P. (2001). Marketing: un enfoque global. México: Mc Graw- Hill , 13a edición.

Redacción. (2008). La evolución del marketing interactivo. Puro marketing, recuperado de: https://www.puromarketing.com/23/4175/evolucion-marketing-interactivo.html.

Redacción. (2013). Marketing de contenido. recuperado de: https://marketingdecontenidos.com/.

Thompson, I. (2006). Definición de marketing. recuperado de: http://www.marketingfree.com/marketing/definicion-marketing.html.

Tirado, D. M. (2013). Fundamentos de Marketing. Castellón de la Plana: Publicaciones de la Universidad de Jaumé. 